\title{
NUEVOS TRILOBITES DEL CÁMBRICO MEDIO DE LAS CADENAS IBÉRICAS (NE ESPAÑA)
}

\author{
Javier ÁLVARO \\ Dpto. Ciencias de la Tierra, Facultad de Ciencias, Universidad de Zaragoza, 50.009-Zaragoza.
}

\begin{abstract}
Álvaro, J. 1996. Nuevos trilobites del Cámbrico Medio de las Cadenas Ibéricas (NE de España). [New trilobites from the Middle Cambrian of the Iberian Chains (NE Spain)]. Revista Española de Paleontología, 11 (1), $75-82$. ISSN 0213-6937.
\end{abstract}

\begin{abstract}
One new subgenus and three new species of trilobites from the Middle Cambrian of the Iberian Chains (NE Spain) are defined. The new taxa are: Solenopleuropsis (Manublesia) subgen. n. (type species: $S$. (M.) verdiagana Sdzuy, 1958), Pardailhania morisca sp. n., Solenopleuropsis (Solenopleuropsis) vizcainoi sp. n., and Solenopleuropsis (Solenopleuropsis) jarquensis sp. n. All of them belong to the Subfamily Solenopleuropsinae Thoral, 1947.
\end{abstract}

Keywords: Trilobites, Solenopleuridae, Middle Cambrian, Iberian Chains, Spain.

\section{RESUMEN}

Se definen en este trabajo un nuevo subgénero y tres nuevas especies de trilobites del Cámbrico Medio de las Cadenas Ibéricas (NE España), pertenecientes a la Subfamilia Solenopleuropsinae Thoral, 1947: Solenopleuropsis (Manublesia) n. subgen. (especie tipo: S. (M.) verdiagana Sdzuy, 1958), Pardailhania morisca n. sp., Solenopleuropsis (Solenopleuropsis) vizcainoi n. sp. y Solenopleuropsis (Solenopleuropsis) jarquensis $\mathrm{n} . \mathrm{sp}$.

Palabras clave: Trilobites, Solenopleuridae, Cámbrico Medio, Cadenas Ibéricas, España.

\section{INTRODUCCIÓN}

La distribución estratigráfica de los trilobites de la Subfamilia Solenopleuropsinae Thoral, 1947 ha venido empleándose en las escalas bioestratigráficas propuestas para el Cámbrico Medio, tanto en la Península Ibérica (Sdzuy, 1968, 1971; Liñán y Gozalo, 1986), como en el sur de Francia (Thoral, 1935, 1948; Courtessole, 1973). Si a la rápida evolución de sus especies y a su relativa abundancia en las rocas sedimentarias, se añade su amplia distribución en otras cuencas marinas de esta época (Terranova: Fletcher, 1972; Martin y Dean, 1988. New Brunswick: Skehan et al., 1978. Gales: Lake, 1931. Marruecos: Mansuy, 1922. Turquía: Dean, 1982; Dean et al., 1986; Shergold y Sdzuy, 1984. Alemania: Wurm, 1928; Schmidt, 1942; Sdzuy, 1957. Cerdeña: Rasetti, 1972; Serpagli y Gnoli, 1984. Bohemia: Barrande, 1846; Snajdr, 1958), una mejora en la identificación de los caracteres genéricos y específicos de esta subfamilia, objetivo de este trabajo, redundará en la mejor precisión de la escala bioestratigráfica del Cámbrico Medio, así como su tentativa aplicación a otras cuencas marinas adyacentes.

\section{SITUACIÓN GEOLÓGICA}

Los trilobites estudiados se han recogido en las rocas del Cámbrico Medio de las Cadenas Ibéricas. Éstas se sitúan en el NE de la Península Ibérica (Fig. 1a), constituyendo una cadena alpina de núcleo paleozoico. Los materiales del Cámbrico Medio se sitúan en la Cadena Ibérica Occidental y en el sector más occidental de la Cadena Ibérica Oriental (Fig. 1b). El registro fósil estudiado se sitúa (Fig. 1c) en las Formaciones Murero (Grupo Mesones; Liñán et al., 1992) y Borobia (Grupo Acón; Álvaro, 1995). La primera, con 50 a $250 \mathrm{~m}$ de potencia, constituye una sucesión monótona de lutitas verdes con algún nivel violáceo en su tercio superior, e intercalaciones de nódulos carbonatados. La Formación Borobia, con 0 a $250 \mathrm{~m}$ de potencia, comprende una alternancia de lutitas verdes y areniscas blancas y grisáceas.

Si bien la Formación Murero contiene una amplia variedad de grupos taxonómicos, las intercalaciones lutíticas basales de la Formación Borobia muestran una disminución drástica del registro fósil. Junto al grupo predominante de los trilobites, aparecen proporciones variables de carpoideos y braquiópodos inarticulados (de concha carbonatada y fosfática) y, en menor medida, hyolíthidos y eocrinoideos. El ambiente sedimentario de la primera formación se situó en un medio fangoso de offshore que experimentó la influencia episódica de tormentas, las cuales quedaron registradas en la desarticulación, fragmentación y acumulación bioclástica del registro paleontológico, formando lumaquelas granoclasificadas y de base erosiva. El comienzo de la Formación Borobia marca un incremento en el tamaño de 

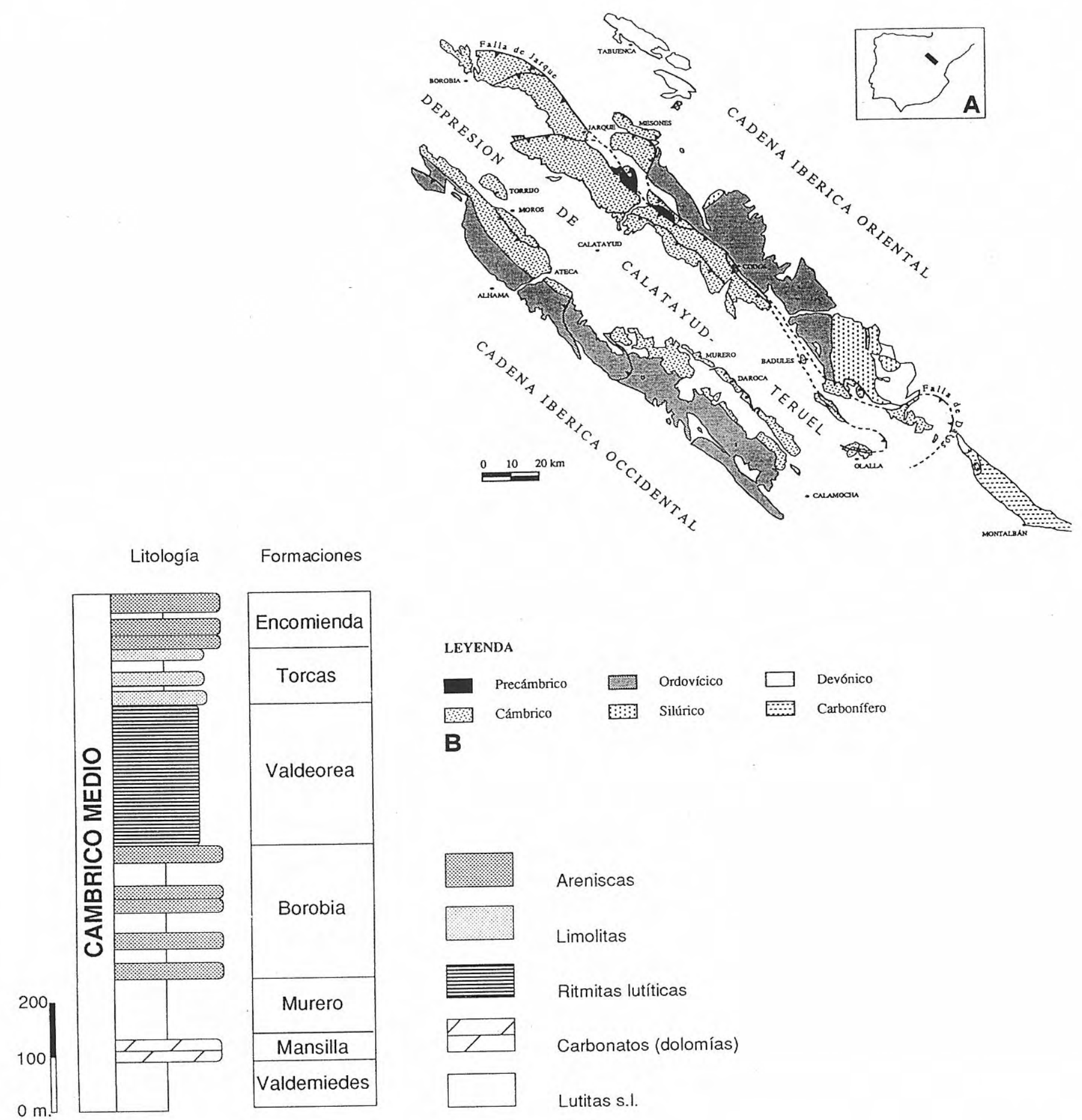

C

Figura 1. (A) Situación de las Cadenas Ibéricas en la Península Ibérica. (B) Afloramientos pre-hercínicos en las Cadenas Ibéricas (tomado de Álvaro, 1995). (C) Unidades litoestratigráficas del Cámbrico Medio de las Cadenas Ibéricas.

grano de los litoclastos, junto al registro de la influencia del oleaje y las corrientes, manifestándose una somerización general gradual en la cuenca (Álvaro, 1994).

\section{PALEONTOLOGÍA SISTEMÁTICA}

La nomenclatura empleada en la descripción taxonómica está basada en los trabajos de Harrington,
Moore y Stubblefield (en Moore, 1959) y Liñán y Gozalo (1986). Los fósiles estudiados en este trabajo se encuentran depositados en el Museo Paleontológico de Zaragoza, marcados con las siglas MPZ.

Para la determinación de los especímenes se han seleccionado únicamente los ejemplares adultos, considerando como tales aquéllos que exhiben un tamaño del cranidio (sag. y/o trans.) superior a $1 \mathrm{~cm}$. Las abreviaturas empleadas son: Lc (longitud del cranidio). 

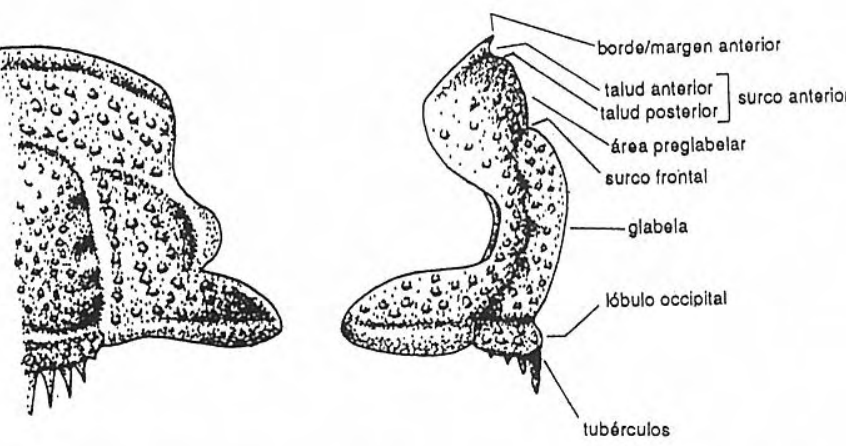

Figura 2. Principales caracteres morfológicos de los cranidios sobre los que se basan las descripciones taxonómicas del trabajo.

Lg (longitud de la glabela), Ac (anchura del cranidio) y Ag (anchura basal de la glabela).

Familia Solenopleuridae Angelin, 1854 Subfamilia Solenopleuropsinae Thoral, 1947

Género Pardailhania Thoral, 1947

Especie tipo: Solenopleura? hispida Thoral, 1935

\section{Pardailhania morisca n. sp.}

Fig. 3, 1-2.

1971 Pardailhania multispinosa Thoral, 1948; Sdzuy, p. 773, tab. 1.

1972 Pardailhania multispinosa Thoral, 1948; Sdzuy, tab. 2.

v.1986 Pardailhania multispinosa Thoral, 1948; Liñán y Gozalo, p. 64; lám. 26, figs. 11-12; lám. 26, figs. 1-3.

Nombre: Referido al gentilicio de la localidad de Moros (Zaragoza), situada en el contacto entre la depresión terciaria de Calatayud-Teruel y los materiales cámbricos de la Cadena Ibérica Occidental.

Holotipo: Cranidio en forma de molde externo, conservado en lutitas margosas (Fig. 3.1). Museo Paleontológico de Zaragoza. MPZ 17.023.

Estrato y localidad tipo: Nivel de unos $7 \mathrm{~m}$ de potencia, situado unos $3 \mathrm{~m}$ por encima de la primera aparición de Pardailhania hispida en la Formación Murero; barranco de Valdemiedes, Murero (Zaragoza). Caesaraugustiense Medio (Cámbrico Medio).

\section{Asociación fósil}

Paradoxides (Eccaparadoxides) rouvillei Miquel, 1905; Pardailhania hispida (Thoral, 1935); Pardailhania multispinosa Thoral, 1948; Agraulos longicephalus (Hicks, 1872); y Conocoryphe (Parabailiella) languedocensis Thoral, 1946.

\section{Material}

28 cranidios y cefalones, conservados en lutitas margosas.

\section{Diagnosis}

Especie del género Pardailhania con tres filas de tubérculos en el área preglabelar; 4 a 6 filas transversales de tubérculos en la glabela, simétricos con respecto a la línea sagital, y que contienen de 2 a 4 tubérculos en cada fila.

Species of the Pardailhania genus showing 3 rows of tubercles in the preglabellar field, and 4 to 6 rows in the glabella.

\section{Descripción}

Cefalón de contorno semicircular, cuya longitud (sag.) equivale aproximadamente a $1 / 2$ de su anchura basal (tr.). Cranidio, de borde anterior arqueado, subtrapezoidal, cuya anchura basal equivale a 1/3 del cefalón y 3/4 o más de su longitud (sag.). Glabela subtrapezoidal a subrectangular, con su parte anterior ligeramente arqueada, moderadamente convexa y con una longitud (sag.) equivalente entre $1 / 2$ y $1 / 3$ de la longitud del cranidio. Área preglabelar abombada. El surco anterior del cranidio es asimétrico, de modo que su talud anterior se prolonga dorso-anteriormente. El borde anterior es tan estrecho que se confunde con el margen anterior. Surco occipital marcado y profundo, delimitando un lóbulo occipital subelíptico. Espinas genales cortas.

Tórax compuesto por 16 a 17 segmentos, con extremidades romas.

La ornamentación está compuesta por tubérculos espiniformes. En la glabela se disponen de 4 a 6 filas transversales de tubérculos, longitudinalmente simétricos, con 1 a 2 tubérculos en cada mitad simétrica. La línea sagital queda a veces insinuada por un posible pasillo longitudinal, donde no se sitúan tubérculos. Éstos tienden a disminuir de tamaño hacia la parte anterior de la glabela. En el área preglabelar se disponen 3 filas de tubérculos (sag.), de menor tamaño o igual que los de la glabela que, ex-sagitalmente, pueden aumentar hasta en dos filas. Arista ocular marcada por la disposición de varios tubérculos. Lóbulo occipital con 1 a 3 filas de tubérculos, en número impar, y con el tubérculo central o espina más desarrollada. Anillos torácicos y pleuras ornamentados con tubérculos.

Dimensiones: El cranidio del ejemplar más grande recolectado alcanza los $25 \mathrm{~mm}$ (sag.); $\mathrm{Lg} / \mathrm{Lc}=7 / 10$ y $\mathrm{Ag} / \mathrm{Ac}=1 / 3$.

\section{Observaciones}

En la amplia descripción que Thoral realizó de las especies Pardailhania hispida (1935) y Pardailhania multispinosa (1948), incluyó la presencia de una y dos filas de tubérculos, respectivamente, en el área preglabelar de los cranidios.

Posteriormente, Sdzuy (1961) define la especie $P$. hispanica, cuyo carácter específico es la presencia, de nuevo, de dos filas de tubérculos en el área preglabelar. Este autor asigna a la especie $P$. multispinosa Thoral, 1948 la presencia de espinas más finas y numerosas que las características de las especies $P$. hispida y $P$. hispanica, pero no cita el número de filas de tubérculos en su área preglabelar. 

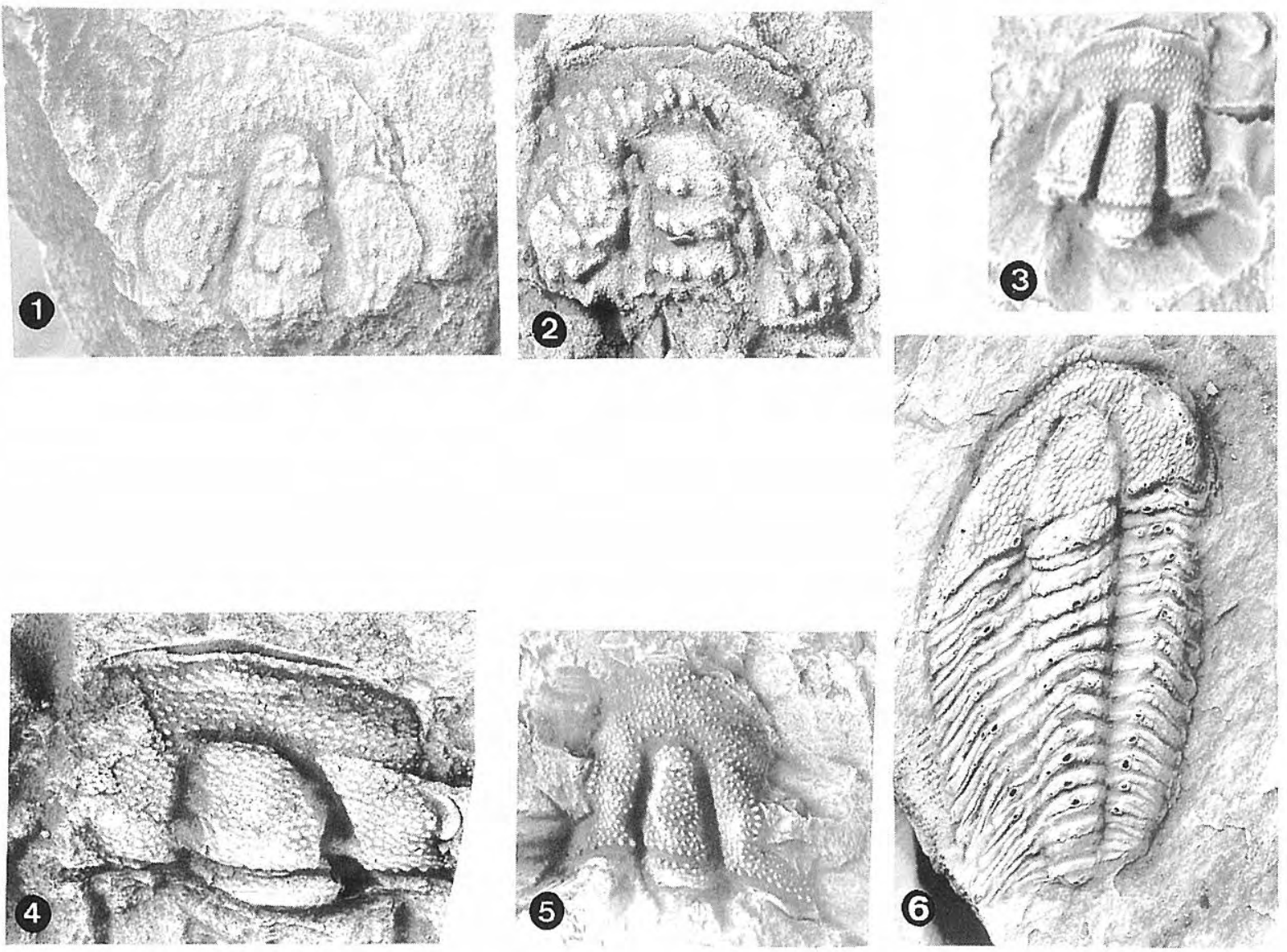

Figura 3. 1.- Pardailhania morisca $n$. sp. Holotipo. Cranidio. Molde externo. x1,5. Murero, RV2/14 (véase Liñán y Gozalo, 1986). MPZ 17023. 2.- Pardailhania morisca n. sp. Paratipo. Cranidio. Molde externo. x1,5. Murero, RV2/14 (véase Liñán y Gozalo, 1986). MPZ 17022. 3.- Solenopleuropsis (Solenopleuropsis) vizcainoi n. sp. Holotipo. Cranidio. Molde externo. x1. Mesones de Isuela. MPZ 17056. 4.- Solenopleuropsis (Solenopleuropsis) vizcainoi $\mathrm{n}$. sp. Paratipo. Cranidio. Molde externo. x1. Mesones de Isuela. MPZ 17057. 5.- Solenopleuropsis (Solenopleuropsis) jarquensis n. sp. Holotipo. Cranidio. Molde externo. x1. Mesones de Isuela. MPZ 17058. 6.- Solenopleuropsis (Solenopleuropsis) jarquensis n. sp. Paratipo. Tórax y cefalón. Molde de látex. x1. Mesones de Isuela. MPZ 17059.

Finalmente, Liñán y Gozalo (1986) asignan a $P$. multispinosa la presencia de tres filas de tubérculos en el área preglabelar.

El hecho de que el holotipo de Pardailhania hispanica posea los caracteres de P. multispinosa Thoral, 1948 y, más concretamente, los de su holotipo, conduce a considerar $P$. hispanica como sinónimo de $P$. multispinosa.

P. morisca se diferencia, por tanto, de P. hispida y $P$. multispinosa en el número de filas de tubérculos en el área preglabelar (que son 1 y 2 respectivamente), y de $P$. sdzuyi en el número de filas de tubérculos glabelares ( 4 a 6 en el caso de $P$. morisca y 8 en $P$. sdzuyi).

\section{Género Solenopleuropsis Thoral, 1947}

\section{Subgénero Solenopleuropsis (Manublesia) n. subgen.}

Especie tipo: Solenopleuropsis verdiagana Sdzuy, 1958

Nombre: Relativo al río Manubles, que discurre desde la sierra del Moncayo (Soria) hasta su desembocadura en el río
Jalón (Ateca, Zaragoza), y cuya erosión ha permitido el acceso a uno de los sectores más fosilíferos de las Cadenas Ibéricas.

\section{Diagnosis}

Subgénero del género Solenopleuropsis que carece de tubérculos en el talud anterior del surco anterior del cranidio (Fig. 2).

Subgenus of Solenopleuropsis showing no tubercles in the anterior slope of the anterior border furrow.

\section{Descripción}

Subgénero del género Solenopleuropsis con el margen anterior del cranidio arqueado o apuntado. Área preglabelar abombada, separando claramente los surcos axial y anterior del cranidio.

Ornamentación del cefalón y tórax compuesta por tubérculos. En la glabela son numerosos, desordenados y con tamaños menores o iguales a los del resto del cefalón. En el área preglabelar se alinean, subconcéntricamente al surco anterior, de 1 a 7 filas de tubérculos. Sus tamaños no son necesariamente uniformes, y se disponen dejando 
posibles "pasillos" entre filas de tubérculos (surcos interpreglabelares sensu Liñán y Gozalo, 1986), subconcéntricos o no al surco anterior del cranidio. Estos pasillos sin tubérculos pueden profundizarse, formando auténticos surcos interpreglabelares e incluso rampas o taludes que diferencian una parte anterior del área preglabelar, ligeramente sobreelevada con respecto a la posterior. El lóbulo occipital presenta de 1 a 5 filas de tubérculos, con número impar de tubérculos en cada fila, destacándose una espina o tubérculo central más desarrollado. La arista ocular aparece insinuada por la alineación de los tubérculos. Borde y talud anteriores del surco anterior del cranidio, sin tubérculos.

Las librígenas poseen varias filas de tubérculos paralelas a la rama anterior de la sutura facial, el lóbulo palpebral y el borde anterior. El resto suele acomodarse uniformemente sin determinar filas netas.

Los anillos torácicos poseen una fila de tubérculos, en número impar, con el central de mayor tamaño. Las pleuras disponen generalmente de una fila de tubérculos, cuyo tamaño disminuye hacia el exterior, y dispuestos todos ellos en $\operatorname{los} 2 / 3$ finales de la pleura.

\section{Método de contaje de las filas de tubérculos}

Como número de filas de tubérculos, se considera en este trabajo al mínimo número contabilizable en el área preglabelar, y no al número de filas de tubérculos apilados en la línea sagital.

\section{Observaciones}

Dado que la especie tipo del género Solenopleuropsis (Conocoryphe rouayrouxi Munier-Chalmas y Bergeron, 1889; en Bergeron, 1889) posee tubérculos en el talud anterior del surco anterior del cranidio, todas las especies incluidas hasta el momento en el género Solenopleuropsis y que carecen de dicho carácter se incluyen ahora en el nuevo subgénero Manublesia; el resto permanece en el subgénero Solenopleuropsis.

Por otro lado, la distribución estratigráfica de ambos subgéneros es sensiblemente diferente. La distribución de las especies del subgénero Manublesia ha venido siendo utilizada en la definición de las biozonas del Caesaraugustiense superior (Sdzuy, 1961; Sdzuy, 1971; Liñán y Gozalo, 1986), mientras que el subgénero Solenopleuropsis aparece únicamente en el Piso sin Solenopleuropsidae de Sdzuy (1971), en el sentido expresado por Gozalo et al. (1994).

\section{Especies}

S. (M.) variolaris (Salter, 1864); S. (M.) ribeiroi Verneuil y Barrande, 1860 (en Prado et al., 1860); S. (M.) verdiagana Sdzuy, 1958; S. (M.) levisilimbata Thoral, 1948; S. (M.) simula Sdzuy, 1958; S. (M.) rubra Sdzuy, 1958; y S. (M.) thorali Sdzuy, 1958.

\section{Subgénero Solenopleuropsis (Solenopleuropsis)} Thoral, 1947

Especie tipo: Conocoryphe rouayrouxi Munier-Chalmas y Bergeron, 1889.

\section{Diagnosis}

Subgénero del género Solenopleuropsis caracterizado por la presencia de tubérculos en el talud anterior del surco anterior del cranidio.

This subgenus is characterized by the presence of tubercles in the anterior slope of the anterior border furrow.

\section{Especies}

S. (S.) multigranifera Thoral, 1948; S. (S.) rouayrouxi (Munier-Chalmas y Bergeron, 1889) (en Bergeron, 1889); S. (S.) vizcainoi n. sp.; y $S$. (S.) jarquensis n. sp.

\section{Edad}

Existe una cierta indeterminación en el límite entre los Pisos Caesaraugustiense (Liñán et al., 1993; sinónimo del Piso de los Solenopleuropsidae, Sdzuy, 1971) y el Piso sin Solenopleuropsidae (Sdzuy, 1971). En su concepción inicial, Sdzuy propuso este último piso del Cámbrico Medio para el ámbito de la Península Ibérica, de modo que no incluyera trilobites fósiles pertenecientes a la Subfamilia Solenopleuropsinae (Solenopleuropsidae sensu Thoral, 1947), situando su límite basal en la desaparición del último ejemplar perteneciente a dicha subfamilia. Incluso, llegó a diferenciar en su parte inferior dos niveles, caracterizándose "por la ausencia de Solenopleuropsis y la abundancia de especies relacionadas con B. levyi" (Sdzuy, 1971; p. 773).

En este trabajo se presentan, por primera vez en la Península Ibérica, nuevos ejemplares pertenecientes al género Solenopleuropsis, en niveles superiores a los descritos por Sdzuy (1971) como Piso de los Solenopleuropsidae, y coincidentes estratigráficamente, con Bailiella levyi. Por tanto, la coexistencia de las especies del subgénero Solenopleuropsis (Manublesia) con $B$. levyi plantea el problema de incluirlas en el Piso "sin Solenopleuropsidae" de Sdzuy.

No obstante, hemos de considerar los datos aportados por Liñán et al. (1993) y Gozalo et al. (1994). Liñán et al. (1993) afirman "The upper limit of the Caesaraugustian Stage is placed at the last record of Solenopleuropsis". Según esta afirmación, el Caesaraugustiense se vería ahora ampliado con los estratos que contienen ejemplares del nuevo subgénero. Sin embargo, Gozalo et al. (1994) consideraron la Biozona de $S$. thorali $(S$. (M.) thorali) como el límite superior del Piso Caesaraugustiense.

Ante esta situación, y como solución transitoria, se emplea en este trabajo la última propuesta (Gozalo et al., 1994) para poder situar sin ambigüedad los ejemplares del nuevo subgénero en la escala bioestratigráfica.

Para solventar este problema, está en preparación un nuevo trabajo en el que se definirá formalmente el último piso del Cámbrico Medio para el ámbito de la Península Ibérica, cuyo límite inferior se delimitará en la primera aparición de una especie de amplia distribución paleogeográfica, y no en la desaparición o extinción de ningún taxón. 
Solenopleuropsis (Solenopleuropsis) vizcainoi $\mathrm{n} . \mathrm{sp}$. Fig. 3, 3-4

\section{p. 1961 Solenopleuropsis simula Sdzuy, 1958; Sdzuy, 645- 647; lám. 26, figs. 1a, 2.}

Nombre: Dedicado a Mr. Daniel Vizcaïno (Carcassonne, Francia), discípulo y continuador de la labor emprendida por el Abad Robert Courtessole en los materiales cambroordovícicos de la Montagne Noire.

Holotipo: Cranidio, conservado como molde externo en lutitas areniscosas (Fig. 3.3). Museo Paleontológico de Zaragoza, MPZ 17056.

Estrato y localidad tipo: Nivel de $4 \mathrm{~m}$ de potencia (visibles, pero limitado superiormente por falla), situado unos $20 \mathrm{~m}$ por encima de la primera aparición de Solenopleuropsis (Manublesia) thorali, en la transición entre las Formaciones Murero y Borobia; Mesones de Isuela (Zaragoza). Piso sin Solenopleuropsidae (Sdzuy, 1971), en el sentido expresado por Gozalo et al. (1994).

\section{Asociación fósil}

Paradoxides (Eccaparadoxides) brachyrhachis Linnarsson, 1883; Paradoxides (Eccaparadoxides)? pradoanus Verneuil y Barrande, 1860; Conocoryphe (Conocoryphe) heberti Munier-Chalmas y Bergeron, 1889; Solenopleuropsis (Manublesia) ribeiroi (Verneuil y Barrande, 1860); Solenopleuropsis (Manublesia) thorali Sdzuy, 1958; Peronopsis ferox (Tulberg, 1880); Bailiella levyi Munier-Chalmas y Bergeron, 1889; y Ctenocephalus coronatus (Barrande, 1846).

\section{Material}

4 cranidios conservados en lutitas samíticas.

\section{Diagnosis}

Especie del subgénero Solenopleuropsis con seis filas de tubérculos en el área preglabelar.

The specific character is the presence of 6 tubercle rows in the preglabellar field.

\section{Descripción}

Cefalón de contorno semicircular, cuya longitud (sag.) equivale aproximadamente a $1 / 2$ de su anchura basal (tr.). Cranidio de borde anterior arqueado, subtrapezoidal, cuya anchura basal equivale a 1/3 del cefalón y $3 / 4$ o más de su longitud (sag.). Glabela subtrapezoidal a subrectangular, con su parte anterior ligeramente arqueada, moderadamente convexa y con una longitud (sag.) equivalente a entre $1 / 2$ y $1 / 3$ de la longitud del cranidio. Área preglabelar abombada, a veces asimétricamente. El surco anterior del cranidio es asimétrico, de modo que su talud anterior se prolonga dorso-anteriormente; a veces es tan tenue que tiende a desaparecer. El borde anterior es tan estrecho que se confunde con el margen anterior. Surco occipital marcado y profundo, delimitando un lóbulo occipital subelíptico. Espinas genales cortas.

Ornamentación del cefalón compuesta por tubérculos. En la glabela son numerosos, desordenados y con tamaños menores o iguales a los del resto del cefalón. En el área preglabelar se alinean, subconcéntricamente al surco anterior, 6 filas de tubérculos. Sus tamaños no son necesariamente uniformes, y se disponen dejando posibles pasillos interpreglabelares (áreas sin tubérculos), subconcéntricos o no al surco anterior del cranidio. Estos pasillos pueden profundizarse, formando auténticos surcos interpreglabelares e incluso rampas o taludes que diferencian una parte anterior del área preglabelar, ligeramente sobreelevada con respecto a la posterior.

Arista ocular insinuada por la alineación de tubérculos. El lóbulo occipital presenta de 3 a 4 filas de tubérculos, con número impar de tubérculos en cada fila, destacándose una espina o tubérculo central más desarrollado. Borde y talud anteriores del surco anterior del cranidio, sin tubérculos.

Los anillos torácicos poseen una fila de tubérculos, en número impar, cuyo tamaño disminuye hacia el exterior, y dispersos todos ellos en los 2/3 finales de las pleuras.

Dimensiones: el cranidio más grande recolectado alcanza (sag.) $20 \mathrm{~mm} ; \mathrm{Lg} / \mathrm{Lc}=2 / 5$ y $\mathrm{Ag} / \mathrm{Ac}=1 / 3$.

\section{Observaciones}

Se diferencia de $S$. (S.) multigranifera y $S$. (S.) rouayrouxi por el número de filas de tubérculos en el área preglabelar.

\section{Solenopleuropsis (Solenopleuropsis) jarquensis n. sp.} Fig. 3, 5-6

p. 1961 Solenopleuropsis simula Sdzuy, 1958; Sdzuy, 645647; lám. 26, fig. 13.

Nombre: Referido a la localidad de Jarque (Zaragoza), situada en la Cadena Ibérica Oriental y en cuyos alrededores se sitúan amplios afloramientos del Cámbrico Inferior y Medio.

Holotipo: Cranidio conservado como molde externo en lutitas areniscosas (Fig. 3.5). Museo Paleontológico de Zaragoza, MPZ 17057.

Estrato y localidad tipo: Nivel de unos $4 \mathrm{~m}$ de potencia (visibles), situado a unos $20 \mathrm{~m}$ por encima de la primera aparición de Solenopleuropsis (Manublesia) thorali, en la transición entre las Formaciones Murero y Borobia; Mesones de Isuela (Zaragoza). Piso sin Solenopleuropsidae (Sdzuy, 1971) en el sentido expresado por Gozalo et al. (1994).

\section{Asociación fósil}

Paradoxides (Eccaparadoxides) brachyrhachis Linnarsson, 1883; Paradoxides (Eccaparadoxides)? pradoanus Verneuil y Barrande, 1860; Conocoryphe (Conocoryphe) heberti Munier-Chalmas y Bergeron, 1889; Solenopleuropsis (Manublesia) ribeiroi (Verneuil y Barrande, 1860); Solenopleuropsis (Manublesia) thorali Sdzuy, 1958; Peronopsis ferox (Tulberg, 1880); Bailiella levyi Munier-Chalmas y Bergeron, 1889; y Ctenocephalus coronatus (Barrande, 1846). 


\section{Material}

Un cranidio y un ejemplar casi completo conservados en lutitas samíticas.

\section{Diagnosis}

Especie del subgénero Solenopleuropsis con siete filas de tubérculos en el área preglabelar.

The specific character is the presence of 7 tubercle rows in the preglabellar field.

\section{Descripción}

Caracteres del cefalón iguales a los de $S$. (S.) vizcainoi n. sp., exceptuando el número de filas de tubérculos en el área preglabelar que, en este caso, es 7.

Dimensiones: El cranidio más grande recolectado alcanza (sag.) $22 \mathrm{~mm} ; \mathrm{Lg} / \mathrm{Lc}=2 / 5$ y $\mathrm{Ag} / \mathrm{Ac}=1 / 3$.

\section{Observaciones}

Se diferencia del resto de especies del subgénero por el número de filas de tubérculos en el área preglabelar.

\section{AGRADECIMIENTOS}

El autor agradece la crítica constructiva realizada por el Prof. K. Sdzuy (Univ. Würzburg) y los Drs. E. Liñán y E. Villas (Univ. Zaragoza), J. García-Alcalde (Univ. Oviedo), A. Perejón (CSIC, Univ. Complutense) y T.P. Fletcher (Geol. Survey, Edinburgh), en el marco de la tesis doctoral donde se incluyó este trabajo, así como a las Dras. I. Rábano y M.L. Martínez Chacón por sus acertados consejos e indicaciones, y al Dr. R. Gozalo por la labor de campo realizada.

Este trabajo constituye una contribución a los I.G.C.P. Projects 319 "Global Palaeogeography of Late Precambrian to Early Palaeozoic" y 336 "Ecological Aspects of the Cambrian Radiation".

\section{BIBLIOGRAFÍA}

Angelin, N.P. 1854. Palaeontologia Scandinavica, Pars II: Crustacea formationis transitionis: fasc. 2, 21-92. Academiae Regiae Scientarum Suecanae (Holmiae). Sampson \& Wallin eds. Stockholm.

Álvaro, J. 1994. El Cámbrico Inferior terminal y Medio de las Cadenas Ibéricas. Bioestratigrafía y Paleogeografía. Tesis Doctoral Univ. Zaragoza, 215 pp. Inédita.

Álvaro, J. 1995. Propuesta de una nueva unidad litoestratigráfica para el Cámbrico Medio-Superior de las Cadenas Ibéricas (NE España): El Grupo Acón. Boletín de la Real Sociedad Española de Historia Natural (Sección Geológica), 90, 95-106.

Barrande, J. 1846. Note préliminaire sur le Système silurien et les Trilobites de Bohême. Leipzig, $250 \mathrm{pp}$.

Bergeron, J. 1889. Étude géologique du Massif ancien situé au Sud du Plateau Central. Annales des Sciences géologiques. (Thèse Faculté de Sciences de Paris), 22, 362 pp. Paris.
Courtessole, R. 1973. Le Cambrien Moyen de la Montagne Noire. Biostratigraphie. Imprim d'Oc, $241 \mathrm{pp}$. Toulouse.

Dean, W.T. 1982. Middle Cambrian Trilobites from the Sosink Formation, Derik-Mardin District, South-Eastern Turkey. Bulletin British Museum (Natural History), Geology Series, 36, 71-81.

Dean, W.T., Monod, O. and Günay, Y. 1986. Lower Paleozoic Stratigraphy in the Southern and Central Amanos Mountains, south central Turkey. Geological Magazine, 123(3), 215-226.

Fletcher, T.P. 1972. Geology and Lower to Middle trilobite faunas of the southern Avalon, Newfoundland. $\mathrm{Ph}$ thesis Univ. Cambridge, 530 pp. Inédita.

Gozalo, R., Liñán, E. y Álvaro, J. 1994. Trilobites de la Subfamilia Solenopleuropsinae THORAL, 1947 del Cámbrico Medio de la Unidad de Alconera (Zona de Ossa-Morena, SO de España). Boletín de la Real Sociedad Española de Historia natural (Sección Geología), 89, 43-54.

Lake, P. 1931. A monograph of the British Cambrian Trilobites. Paleontographical Society Monograph, 350 pp. London.

Liñán, E. y Gozalo, R. 1986. Trilobites del Cámbrico inferior y medio de Murero (Cordillera Ibérica). Memorias del Museo Paleontológico de la Universidad de Zaragoza, 2, 104 pp.

Liñán, E., Gozalo, R., Gámez, J.A. y Álvaro, J. 1992. Las formaciones del Grupo Mesones (Cámbrico InferiorMedio) en las Cadenas Ibéricas. Actas de las Sesiones Científicas III Congreso Geológico de España, 1, $517-$ 523.

Liñán, E., Perejón, A. and Sdzuy, K. 1993. The LowerMiddle Cambrian stages and stratotypes from the Iberian Peninsula: a revision. Geological Magazine, 139(6), 817833.

Mansuy, H. 1922. Déscription de quelques trilobites du Cambrien du Maroc. Bulletin Société géologique France, 22, 285-290.

Martin, F. and Dean, W.T. 1988. Middle and Upper Cambrian Acritarch and Trilobite Zonation at Manuels River and Random Island, Eastern Newfoundland. Geological Survey Canada Bulletin, 381, 54 pp.

Moore, R. (Ed.) 1959. Treatise on Invertebrate Paleontology. Part 0. Arthropoda 1. Geological Society American and Kansas University Press. Lawrence, 560 pp.

Prado, C. de, Verneuil, E. et Barrande, J. 1860. Sur l'existence de la faune primordial dans la Chaîne Cantabrique. Bulletin Société géolique France, série 2, 17, 516-542.

Rasetti, F. 1972. Cambrian Trilobite Faunas of Sardinia. Atti della Academia Nazionale dei Lincei, Memorie, 11(1), 100 pp. . Roma.

Salter, J.W. 1864. On some new fossils from the Lingulaflags of Wales. Quarterly Journal Geological Society of London, 19, 233-241.

Schmidt, W.E. 1942. Die mittelkambrische Fauna von Doberlug. Jarhbuch Reichsstelle Bodenforsch, 62, 344402.

Sdzuy, K. 1957. Revision der mittlekambrischen Trilobiten von Doberlug. Senckenbergiana lethaea, 38(1-2). 7-28. 
Sdzuy, K. 1958. Neue Trilobiten aus dem Mittelkambrium von Spanien. Senckenbergiana lethaea, 39(3/4), 235254.

Sdzuy, K. 1961. Das Kambrium Spaniens. Teil II. Trilobiten. Akademie Wissenschaften und der Literatur Mainz Abhandlungender Mathematisch Naturwissenschaftlichen Klasse, 7(8), 217-408.

Sdzuy, K. 1971. La subdivisión bioestratigráfica y la correlación del Cámbrico medio de España. I Congreso Hispano-Luso-Americano de Geología Económica II (Sección Geológica), 2, 769-782.

Sdzuy, K. 1972. Das Kambrium der Acadobaltischen Faunenprovinz. Zentralblatt für Geologie und Paläontologie, 2(1), 1-91.

Serpagli, E. and Gnoli, M. 1984. Palaeozoic Palaeontology in Sardinia: a review (1857-1983). Bollettino del Museo Regionale di Scienze Naturali, Torino, 2(1), 163-180.

Shergold, J.H. and Sdzuy, K. 1984. Cambrian and early Tremadocian trilobites from Sultan Dag, central Turkey. Senckenbergiana lethaea, 65(1/3), 51-135.
Skehan, J.W., Murray, D.P., Palmer, A.R., Smith, A.T. and Belt, E.S. 1978. Significance of fossiliferous Middle Cambrian rocks of Rhode Island to the history of the Avalonian microcontinent. Geology, 6, 694-698.

Snajdr, M. 1958. Trilobiti ceského stredního kambria. Razpravy Ustredního ústavu geologického, 24, 1-280.

Thoral, M. 1935. Contribution à l'étude paléontologique de l'Ordovicien inférieur de la Montagne Noire et Révision sommaire de la faune cambrienne de la Montagne Noire. Imprimerie de la Charité, 362 pp. Montpellier.

Thoral, M. 1947. Note sur quelques fossiles acadiens du NW de l'Espagne. Annales Société géologique du Nord, 67, 52-81.

Thoral, M. 1948. Solenopleuridae et Liostracidae languedocensis. Annales Université Lyon, Sciences Naturelles, 5, 1-89.

Wurm, A. 1928. Ueber eine neue mittelcambrische Fauna aus dem bayrischen Frankenwald und ihre Bedeutung für die Stratigraphie des älteren Paläozoicums. Neues Jahrbuch für Mineralogie, 59(B), 31-47.

Manuscrito recibido: 27 de enero, 1995 Manuscrito aceptado: 30 de mayo, 1995 